ISSN 0103-5150

Fisioter. Mov., Curitiba, v. 26, n. 2, p. 403-411, abr./jun. 2013

Licenciado sob uma Licença Creative Commons

\title{
Adaptações crônicas sobre a função pulmonar em indivíduos infectados pela leishmaniose visceral
}

\author{
Chronic adaptations on lung function in individuals \\ infected with Visceral Leishmaniasis
}

\author{
Andrea Oliveira da Silva ${ }^{[a]}$, Sandra Brito Silva ${ }^{[b]}$, Caio Breno Reis Pires ${ }^{[c]}$, \\ Luciana Soares Lages Gonçalves $^{[\mathrm{d}]}$, Patrícia Chaves Coertjens ${ }^{[\mathrm{e}]}$, Marcelo Coertjens ${ }^{[\mathrm{f}]}$ \\ [a] Fisioterapeuta, curso de Fisioterapia da Universidade Federal do Piauí (UFPI), Parnaíba, PI - Brasil, e-mail: \\ andrea.phb@hotmail.com \\ [b] Fisioterapeuta, curso de Fisioterapia da Universidade Federal do Piauí (UFPI), Parnaíba, PI - Brasil, e-mail: \\ san_fisio@hotmail.com \\ [c] Fisioterapeuta do Hospital Santa Edwirges, Parnaíba, PI - Brasil, e-mail: caiobreno@uol.com.br \\ [d] Médica do Hospital Santa Edwirges, Parnaíba, PI - Brasil, e-mail: luciannalages@yahoo.com \\ [e] Professor assistente do curso de Fisioterapia da Universidade Federal do Piauí (UFPI), Parnaíba, PI - Brasil, e-mail: \\ paticoertjens@hotmail.com \\ [f] Professor assistente do curso de Fisioterapia da Universidade Federal do Piauí (UFPI), Parnaíba, PI - Brasil, e-mail: \\ coertjens@hotmail.com
}

\section{Resumo}

Introdução: Apesar de escassas, as principais alterações cardiopulmonares verificadas durante a infecção com Leishmaniose Visceral (LV) se referem a alterações histológicas e imunológicas e poucos relatos de repercussões funcionais. Objetivo: Verificar e analisar a existência de adaptações crônicas na função pulmonar de indivíduos assintomáticos previamente infectados pela LV. Materiais e métodos: Trata-se de um estudo do tipo transversal descritivo, para o qual foram selecionados todos os indivíduos que contraíram LV em Parnaíba (Piauí, Brasil) entre 2007 e 2010 de acordo com informações do Setor de Epidemiologia do município. De um total de 20 indivíduos com confirmação parasitológica de LV, foram avaliados nove com idades entre 18 e 62 anos entre setembro e outubro de 2011. Excluíram-se indivíduos que não apresentaram diagnóstico parasitológico de LV, doenças ou sintomas cardiorrespiratórios recentes, usuários crônicos de drogas ilícitas e gestantes. Foram realizadas medidas antropométricas e testes de espirometria e de manovacuometria conforme 
especificações da American Thoracic Society / European Respiratory Society (ATS/ERS). Para comparação das médias dos valores medidos e preditos foi realizado Teste $t$ Student para dados dependentes. 0 nível de significância adotado foi p < 0,05. Resultados: A Capacidade Vital Forçada (CVF) $(3,8 \pm 0,9$ vs. 4,2 $\pm 0,8$ L) e o Volume Expiratório Forçado no primeiro segundo $\left(\mathrm{VEF}_{1}\right)(3,3 \pm 0,7$ vs. 3,5 \pm 0,7 L) foram estatisticamente menores do que os valores medidos ( $\mathrm{p}<0,01)$. A relação $\mathrm{VEF}_{1} / \mathrm{CVF}(87,6$ vs. 84,4\%) foi maior nos valores medidos ( $\mathrm{p}=0,08)$. Conclusão: Apesar de o número de indivíduos avaliados ser pequeno, os dados indicam que a infecção por LV parece desenvolver adaptações crônicas sobre o sistema respiratório, resultando em volumes pulmonares significativamente diminuídos e uma tendência a padrões respiratórios restritivos.

Palavras-chave: Leishmaniose Visceral. Sistema respiratório. Testes de função pulmonar.

\section{Abstract}

Introduction: Although scarce, the main changes observed during cardiopulmonary infection with Visceral Leishmaniasis (VL) refer to histological and immunological and few reports of functional repercussions. Objective: To verify and analyze the existence of chronic adaptations in pulmonary function in individuals who have been infected by VL. Materials and methods: It is a descriptive cross-sectional study, which evaluated nine people aged between 18 and 62 years. Anthropometric measurements and spirometry and manometer tests were performed according to the specifications of the American Thoracic Society / European Respiratory Society (ATS/ERS). For comparison of the measured and predicted values was performed Student $t$ test for dependent data. The level of significance was $p<0.05$. Results: The Forced Vital Capacity (FVC) $(3,8 \pm 0,9 \pm 0,8$ vs. 4,2 I) and Forced expiratory volume in first second $\left(F E V_{1}\right)(3,3 \pm 0,7 \pm 0,7 \mathrm{vs} .3,5 \mathrm{l})$ were statistically smaller in measured values $(p<0.05)$. The $F E V_{1} / F V C$ ratio (87.6 vs. 84.4\%) was higher in the measured values ( $\left.p=0.08\right)$. Conclusion: The data indicate that infection with VL seems to develop chronic adaptations on the respiratory system, resulting in decreased lung volumes and a tendency to restrictive breathing patterns.

Keywords: Visceral Leishmaniasis. Respiratory system. Pulmonary function tests.

\section{Introdução}

A Leishmaniose Visceral (LV) é uma doença parasitária de alta incidência mundial, sendo atualmente considerada um problema grave de saúde pública (1). No continente americano, $90 \%$ dos casos encontram-se no Brasil, principalmente, na região Nordeste e seus primeiros relatos datam desde o ano de $1934(2,3)$. Historicamente negligenciada pelo setor privado, se caracteriza, principalmente, por atingir a população de classe média baixa e da zona rural. Entretanto, nas últimas décadas, vêm sofrendo um processo de urbanização (4), verificada com base no surgimento de surtos epidêmicos em grandes centros urbanos, o que a tornou uma das prioridades da Organização Mundial da Saúde (OMS) dentre as doenças tropicais $(5,6,7)$. A LV é uma doença grave que atinge todas as faixas etárias e, quando não tratada, seu índice de morte pode chegar a $95 \%(8)$.

A LV no Brasil tem como principal agente etiológico o parasito leishmania chagasi, cuja transmissão ocorre pela picada do inseto flebotomíneo em animais silvestres como raposa e marsupiais ou em animais de ambiente doméstico, sendo o cão o principal hospedeiro $(5,9)$. Com a picada, o inseto vetor ingere parasitos que estejam na pele ou no sangue do animal contaminado, podendo inoculá-los no próximo animal ou no ser humano que for picado, os quais irão desenvolver, consequentemente, a infecção (10).

Sua principal ação no organismo consiste na instalação de um infiltrado inflamatório mononuclear obtido pela reprodução do parasita dentro dos macrófagos do hospedeiro, causada por uma ineficácia das citocinas produzidas pelas células Th1, TCD4+ e TCD8+ em neutralizar o parasita $(11,12)$. Após penetração, a leishmania sofre diferenciação quanto à sua forma de promastigota para amastigota e se multiplica intensamente até o rompimento dos macrófagos (13). Com isso, elas são liberadas para serem, novamente, fagocitadas num processo contínuo, desenvolvendo então à disseminação hematogênica para outros tecidos como linfonodos, fígado, baço e 
medula óssea $(13,14)$. Nesse processo, órgãos como, rins, pulmão, coração e, principalmente, fígado e baço podem ser infectados. Os sintomas irão variar de acordo com o órgão afetado e a carga parasitaria no local (15). Quando instalado, ocasiona, entre outras manifestações, febre irregular por tempo prolongado, severo emagrecimento, intensa palidez cutâneo-mucosa, além de icterícia, hepatoesplenomegalia, anemia, leucopenia e trombocitopenia (16).

Acometimentos cardiopulmonares são considerados raros tanto em animais como em humanos. Em cães, existem relatos de modificações do ritmo cardíaco, poliarterite necrosante, granulomas, miocardite não supurativa e multifocal acompanhada por necrose e degeneração das fibras miocárdicas, além de dispneia, rinite, corrimento nasal, epistaxe, ausculta anormal, pneumonia intersticial crônica e difusa com infiltrado linfoplasmocitário $(15,17,18)$. Em humanos, relatos de casos descrevem que dentre as principais alterações em pacientes com envolvimento pulmonar estão a pneumonite intersticial (19, $20)$ e o derrame pleural $(21,22)$. Outros sintomas comumente encontrados são a tosse seca e, eventualmente, dispneia (23), inflamação generalizada da árvore brônquica (24), além da pneumocitys carini (25). Outro aspecto importante que tem sido verificado é a relação de coinfecção HIV/LV (26), com relatos de aparecimento de nódulo pulmonar solitário (19), como, também, infiltrações pulmonares (27) e adenoma bronquioalveoloar (28). Além disso, alterações cardíacas como diminuição na altura das ondas T, ondas T invertidas, segmento ST côncavo e prolongamento do intervalo QT corrigido são verificadas após o tratamento desses pacientes com antimoniais por causa de cardiotoxicidade provocada por esses medicamentos (29). Embora raros, ainda existem relatos de morte súbita desencadeada em decorrência dessa toxicidade (30).

Além de escassas, as principais alterações cardiopulmonares verificadas durante a infecção com LV se referem, principalmente, a alterações histológicas e imunológicas em cães ou durante a coinfecção HIV/ $\mathrm{LV}$, consistindo, principalmente, de relatos de casos desprovidos das repercussões funcionais desses órgãos. Existe, portanto, uma lacuna envolvendo as características funcionais do sistema cardiorrespiratório em indivíduos infectados com LV. Sabemos que adaptações estruturais cardíacas e pulmonares podem desencadear profundas alterações funcionais nos órgãos acometidos. Nesse sentido, o objetivo do nosso estudo foi verificar a existência de adaptações crônicas na função pulmonar em indivíduos assintomáticos que foram infectados pela LV no município de Parnaíba (PI).

\section{Materiais e métodos}

Trata-se de um estudo transversal descritivo, cujo objetivo foi avaliar a repercussão na função pulmonar em indivíduos previamente diagnosticados com LV e que estivessem livres dos seus sintomas após tratamento medicamentoso. De acordo com dados fornecidos pelo Setor de Epidemiologia da Secretaria de Saúde do município de Parnaíba (PI), os indivíduos de ambos os sexos com faixa etária entre 18 e 65 que foram diagnosticados com $\mathrm{LV}$ no período compreendido entre 2007 e 2010, neste município, totalizaram 20 pacientes. Desse total, apenas 15 foram localizados, visto que três não residiam nos endereços fornecidos e dois foram a óbito. Os pacientes localizados foram convidados a responder um formulário adaptado a partir do Questionário Respiratório proposto por Pereira (31), composto por uma anamnese do paciente incluindo hábitos de vida, questionamentos sobre a confirmação do diagnóstico da LV, bem como a existência de doenças respiratórias previamente diagnosticadas.

Os critérios de inclusão avaliados no formulário foram: indivíduos de ambos os sexos com idade entre 18 e 65 anos e que tinham sido infectados pela LV nos últimos quatro anos com diagnóstico confirmado por exames parasitológicos. Os critérios de exclusão foram: indivíduos que não tinham diagnóstico confirmado de LV através de exames parasitológicos, que apresentavam quadro agudo de tuberculose, bronquite crônica, asma, edema pulmonar, enfisema pulmonar, infecção respiratória, hemoptise, angina recente, descolamento da retina, crise hipertensiva e alterações estruturais avançadas que comprometem a função ventilatória, tais como escoliose, cifoescoliose e outras, usuários crônicos de drogas ilícitas e indivíduos no período gestacional (31). De acordo com a análise desses formulários, constatou-se que dois indivíduos eram usuários crônicos de drogas ilícitas, um encontrava-se com tuberculose e um encontrava-se em período gestacional, sendo estes excluídos da pesquisa. Dessa forma, foram selecionados para a pesquisa 11 pacientes. Dos indivíduos selecionados, dois se recusaram participar do estudo, restando nove participantes para a realização das avaliações. 
Um cálculo do tamanho da amostra foi realizado previamente assumindo uma variância e uma diferença entre a média da amostra e a média verdadeira (erro) estimadas em um litro nos valores de espirometria e nível de significância $(t \alpha)$ de 0,05 . Apesar de a quantidade de indivíduos avaliados (nove) ter sido menor do que mínimo estimado (doze), foi possível detectar diferenças estatisticamente significativas nas variáveis analisadas. Todos os indivíduos receberam orientação individual sobre os objetivos e procedimentos deste estudo, bem como, assinaram Termo de Consentimento Livre e Esclarecido aprovado pelo Comitê de Ética da Universidade Federal do Piauí (CAAE n. 0298.0.045.000-11), estabelecido pela Resolução n. 196/1996 sobre pesquisa envolvendo seres humanos que atendem a exigências éticas e científicas fundamentais.

Os indivíduos selecionados foram orientados a: não ingerir café, chá preto ou qualquer outro tipo de substância estimulante seis horas antes dos testes; não ingerir bebidas alcoólicas e não consumir tabaco quatro horas antes dos testes; e evitar refeições volumosas duas horas antes dos testes. Os sujeitos foram orientados também a não realizar nenhuma outra atividade física intensa ou exercícios físicos nas 48 horas anteriores às coletas (31). Foram realizados avaliação da estatura e massa corporal e testes de espirometria e de manovacuometria, conforme especificações da ATS/ERS (32). Para a realização de ambos os testes, os indivíduos tiveram feedback visual e receberam incentivo verbal padronizado pelo mesmo avaliador.

Para realização dos testes espirométricos, foi utilizado um espirômetro portátil (MicroQuark; Cosmed, Roma, Itália), cuja calibração foi realizada manualmente com uso de uma seringa de três litros conforme orientação do fabricante. Os parâmetros espirométricos avaliados, bem como seus respectivos valores preditos (33), foram: Capacidade Vital Forçada (CVF), Volume Expiratório Forçado no primeiro segundo $\left(\mathrm{VEF}_{1}\right)$, razão entre Volume Expiratório Forçado no primeiro segundo e Capacidade Vital Forçada $\left(\mathrm{VEF}_{1} / \mathrm{CVF}\right)$, Pico de Força Expiratória (PFE), e Fluxo Expiratório Forçado entre 25 e $75 \%$ da CVF $\left(\mathrm{FEF}_{25-75 \%}\right)$. As manobras para a realização do teste foram repetidas no mínimo três vezes, selecionando-se o maior valor obtido. Foram consideradas apenas as provas reprodutíveis com variação inferior a 5\%. 0 indivíduo, sentado, era orientado a inspirar até atingir a Capacidade Pulmonar Total (CPT) e, em seguida, encorajado a expirar até o Volume Residual
(VR) através de uma boquilha (32). Em todas as provas os indivíduos fizeram uso de clipe nasal.

Para a avaliação da força muscular respiratória, os sujeitos foram submetidos ao teste de manovacuometria (MVD 300; GlobalMed, Porto Alegre, Brasil) cuja calibração foi realizada automaticamente conforme orientação do fabricante. Os parâmetros avaliados foram Pressão Inspiratória Máxima (PImáx) e Pressão Expiratória Máxima (PEmáx). Para a avaliação de PImáx, os indivíduos permaneciam na posição sentada e, utilizando um clipe nasal, eram orientados a realizar um esforço inspiratório máximo a partir do VR. Para a avaliação da PEmáx, os indivíduos foram orientados a realizar um esforço expiratório máximo a partir da CPT. Todos os participantes realizaram no mínimo três manobras reprodutíveis, cada uma mantida por pelo menos 1,5 segundos (32). Para análise dos dados, o valor mais alto era registrado, contanto que não excedesse em $20 \%$ o segundo valor mais alto do indivíduo. Os valores obtidos foram comparados aos valores de normalidade para a população brasileira, sendo o resultado expresso em percentagem (\%PImáx e \%PEmáx), permitindo a classificação dos pacientes com musculatura respiratória adequada, quando a PImáx e PEmáx fosse maior ou igual a 75\% do previsto para homens e mulheres de 20 a 80 anos de acordo com Neder et al. (34). Curvas e ou valores inadequados obtidos nos testes de função pulmonar foram descartados da amostra.

Os dados foram analisados por meio de estatística descritiva (média e desvio padrão). A normalidade dos dados foi avaliada pelo teste de Shapiro-Wilk (Lilliefors) ( $p>0,05)$. Para comparação das médias intragrupos (valores medidos vs. valores preditos), utilizou-se um Teste $t$ Student para dados dependentes. 0 nível de significância adotado foi $\mathrm{p}<0,05$. 0 pacote estatístico utilizado foi o SPSS, versão 11.0.

\section{Resultados}

A partir da análise do formulário, constatou-se que: em relação ao sexo, 78\% dos indivíduos ( $n=7$ ) eram do sexo masculino; $33,3 \%$ dos pacientes $(\mathrm{n}=3)$ avaliados manifestaram tosse durante o período de infecção por LV; e 22,2\% (n = 2) apresentaram dispneia no mesmo período. No entanto, sintomas de tosse ou dispneia não foram relatados durante o período das coletas de dados. Observou-se, também, que $33,3 \%$ dos indivíduos $(\mathrm{n}=3)$ fumaram por mais 
de 10 anos e que haviam parado depois do período de infecção e $11,1 \%(n=1)$ continuavam fumando. Além disso, um indivíduo estava infectado pelo vírus HIV e 22,2\% ( $n=2)$ desenvolveram asma brônquica e tuberculose durante a vida.

Todas as variáveis avaliadas apresentaram normalidade ( $\mathrm{p}>0,05)$. As médias de idade, massa, estatura, e IMC foram respectivamente $34,2 \pm 13,5$ anos; $72 \pm$ $16,3 \mathrm{~kg} ; 162 \pm 0,1 \mathrm{~cm}$ e $27,3 \pm 6,2 \mathrm{~kg} / \mathrm{m}^{2}$. Em relação aos testes de espirometria, a análise dos dados demonstrou que a CVF e o $\mathrm{VEF}_{1}$ apresentaram diferenças estatisticamente significativas entre valores medidos e previstos $(\mathrm{p}<0,01)$. ACVF apresentou médias de 3,8 $\pm 0,9 \mathrm{~L}$ para os valores medidos e 4,2 $\pm 0,8 \mathrm{~L}$ para os valores previstos e no $\mathrm{VEF}_{1}$ as médias foram $3,3 \pm 0,7 \mathrm{~L}$ para os valores medidos e $3,5 \pm 0,7 \mathrm{~L}$ para os valores previstos. A relação $\mathrm{VEF}_{1} / \mathrm{CVF}$ foi de $87,6 \pm 4,2 \%$ para os valores medidos e $84,4 \pm 2,0 \%$ para os valores previstos ( $\mathrm{p}=0,08)$. As variáveis $\mathrm{PFE}_{\text {e }} \mathrm{FEF}_{25-75 \%}$ e as variáveis de pressões respiratórias não apresentaram diferenças significativas (Tabela 1 e Gráfico 1).

\section{Discussão}

Por meio da análise dos dados obtidos a partir do formulário foi constatado o surgimento de sinais clínicos respiratórios durante a contaminação pelo parasita em $44,4 \%$ dos pacientes ( $n=4)$, dentre eles a tosse e a dispneia, evidenciando a presença do envolvimento respiratório na LV. Reforçando os nossos resultados, Marshall et al. (24) descrevem a tosse e a dispneia em seu estudo de caso, enquanto que Herrejon et al. (23) relatam episódios de tosse como sintoma comumente encontrado na LV. Durante a infestação pela LV, a reprodução do parasita acarreta aumento dos órgãos toracoabdominais afetados, provocando uma distensão abdominal considerável (35). Esse aumento do volume abdominal provavelmente ocasionará adaptações na mecânica respiratória, originando sintomas respiratórios tais como dispneia e tosse $(36,37)$. Com base nesses achados clínicos e nas alterações estruturais proporcionadas pela infecção, foram realizadas provas de função pulmonar em pacientes acometidos previamente por LV.

Em relação à força muscular respiratória avaliada mediante o teste de manovacuometria, não foram encontradas diferenças significativas entre os valores medidos e previstos nos valores de PImáx e PEmáx. Entretanto, 33,3\% da população estudada não alcançou o índice de $75 \%$ do valor previsto para a PImáx de acordo com Neder et al. (34), caracterizando fraqueza da musculatura inspiratória. Esses resultados podem ter influenciado na redução de cerca de $15 \mathrm{cmH}_{2} \mathrm{O}$ nas médias de PImáx medida em comparação com a prevista (Tabela 1).

Tabela 1 - Média, desvio padrão e valores mínimos e máximos dos valores de funções pulmonares medidos e previstos nos indivíduos assintomáticos previamente infectados por Leishmaniose Visceral (LV) na cidade de Parnaíba (PI), 2011

\begin{tabular}{|c|c|c|c|c|c|c|}
\hline \multirow[b]{2}{*}{ Funções Pulmonares } & \multicolumn{3}{|c|}{ Valores Medidos } & \multicolumn{3}{|c|}{ Valores Previstos } \\
\hline & média \pm d.p. & Mín. & Máx. & média \pm d.p. & Mín. & Máx. \\
\hline PEmáx $\left(\mathrm{cmH}_{2} \mathrm{O}\right)$ & $136,3 \pm 35,0$ & 75,0 & 205,0 & $131,3 \pm 22,7$ & 86,9 & 150,7 \\
\hline PImáx $\left(\mathrm{cmH}_{2} \mathrm{O}\right)$ & $105,4 \pm 27,4$ & 75,0 & 159,0 & $120,5 \pm 19,1$ & 87,3 & 140,9 \\
\hline CVF (L) & $3,8 \pm 0,9$ & 2,1 & 4,8 & $4,2 \vee 0,8^{a}$ & 2,9 & 5,4 \\
\hline $\operatorname{VEF}_{1}(\mathrm{~L})$ & $3,3 \pm 0,7$ & 1,9 & 4,2 & $3,5 \pm 0,7^{\mathrm{a}}$ & 2,4 & 4,5 \\
\hline $\mathrm{VEF}_{1} / \mathrm{CVF}(\%)$ & $87,6 \pm 4,2$ & 81,7 & 95,5 & $84,4 \pm 2,0$ & 81,7 & 88,8 \\
\hline PEF (L/min.) & $473,9 \pm 141,4$ & 280,4 & 753,5 & $495,5 \pm 87,7$ & 373,5 & 603,6 \\
\hline $\mathrm{FEV}_{25-75 \%}(\mathrm{~L} / \mathrm{s})$ & $4,0 \pm 1,1$ & 2,5 & 5,6 & $4,2 \pm 0,7$ & 2,9 & 5,3 \\
\hline
\end{tabular}

Legenda: PEmáx = pressão expiratória máxima; PImáx = pressão inspiratória máxima expressa em valores absolutos, desprezando-se 0 sinal de negatividade; $C V F=$ capacidade vital forçada; $\mathrm{VEF}_{1}=$ volume expiratório forçado no primeiro segundo, $V E F_{1} / C V F=$ razão entre volume expiratório forçado no primeiro segundo e capacidade vital forçada, $\mathrm{PEF}=$ pico de fluxo expiratório e $\mathrm{FEV}_{25-75 \%}=$ fluxo expiratório forçado entre 25 e $75 \%$ da CVF; $a=p<0,01$.

Fonte: Dados da pesquisa. 


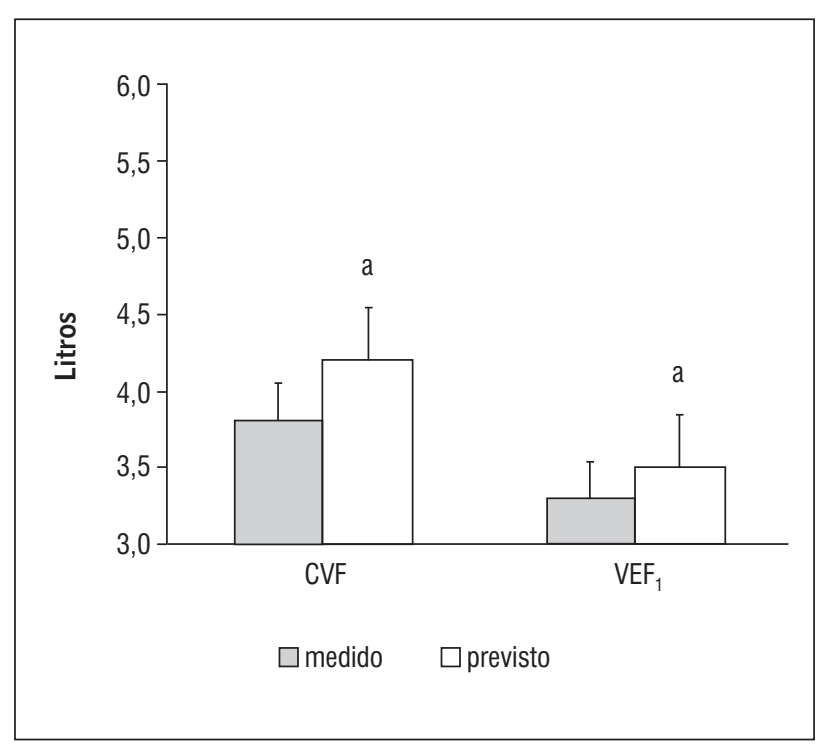

Gráfico 1 - Valores medidos e previstos de capacidade vital forçada (CVF) e volume expiratório forçado no primeiro segundo (VEFI) de indivíduos assintomáticos previamente infectados por Leishmaniose Visceral (LV) na cidade de Parnaíba (PI), 2011

Legenda: $a=p<0,01$.

Fonte: Dados da pesquisa.

As médias dos valores medidos de CVF e do $\mathrm{VEF}_{1}$ foram significativamente menores em comparação a dos valores previstos (Gráfico 1). Esses resultados demonstram que os indivíduos que sofreram infecção por LV podem apresentar, posteriormente, alterações funcionais no sistema respiratório.

Estudos distintos têm avaliado as repercussões na função pulmonar durante a infecção de diferentes parasitoses. Por exemplo, diminuição na CVF e na CPT foi encontrada em pacientes infectados agudamente por esquistossomose mansônica na forma hepatoesplênica (38). Também foi encontrada uma discreta diminuição do $\mathrm{VEF}_{1}$ em indivíduos com Mal de Chagas portadores de cardiopatias, esofagopatias e colopatias, quando comparados ao $\mathrm{VEF}_{1}$ dos indivíduos normais (39). Entretanto, na análise de espirogramas de 15 pacientes com Mal de Chagas não foram observadas alterações pulmonares funcionais significativas (40). Em outro estudo, de um total de 62 indivíduos com Malária, 47\% dos pacientes apresentaram sintomas respiratórios, 53,2\% tiveram alterações espirométricas e $63 \%$ manifestaram alteração na gasometria arterial, demonstrando assim um relativo comprometimento pulmonar em indivíduos com tal parasitose (41). Avaliando a função pulmonar da espirometria de 35 pacientes com paracoccidioidomicose, $50 \%$ deles manifestaram padrão respiratório normal, $57 \%$ do tipo obstrutivo e apenas 8\% distúrbio ventilatório do tipo misto (42).

Em nosso estudo, a relação $\mathrm{VEF}_{1} / \mathrm{CVF}$ medida alcançou média de $87,6 \%$, enquanto que a relação prevista alcançou média de $84,4 \%$. Apesar de as médias dos valores encontrarem-se dentro da normalidade, os valores medidos estão próximos do limite da classificação restritiva. Analisando-se os dados individualmente, quatro sujeitos desta amostra apresentaram $\mathrm{VEF}_{1} / \mathrm{CVF}$ superior a $90 \%$ e três obtiveram seus valores acima do previsto - próximo aos $90 \%$. Esse resultado se justifica pelo fato de a redução na CVF ter sido superior à redução verificada na $\mathrm{VEF}_{1}$, característico do padrão respiratório restritivo. Tal distúrbio ventilatório restritivo é verificado em doenças pulmonares intersticiais, em que, semelhantemente à infecção por LV, ocorre um acúmulo de macrófagos no interstício resultando cronicamente no desenvolvimento de fibrose do parênquima pulmonar, reduzindo, dessa forma, os volumes pulmonares $(43,44)$. Diversos estudos sobre a LV têm relatado o desenvolvimento do infiltrado intersticial tanto em humanos $(19,20)$ como em cães $(18,45)$. Duarte et al. (45) analisaram os achados histopatológicos de 41 cães infectados por LV, detectando a pneumonite intersticial em $81,5 \%$ dos animais.

Padrões respiratórios restritivos têm sido encontrados em outras doenças parasitárias. Durante a avaliação de 30 pacientes portadores de esquistossomose hepatoesplênica, constatou-se, a partir dos espirogramas, que $30 \%$ dos esquistossômoticos apresentavam padrão ventilatório restritivo (46). Esses resultados são semelhantes àqueles encontrados em doenças tipicamente pulmonares. Por exemplo, Park et al. (47) verificaram a espirometria de 21 indivíduos com doença pulmonar intersticial associada a bronquiolite e observaram que $38 \%$ da população estudada demonstraram valores considerados normais, $35 \%$ apresentaram padrão ventilatório restritivo e $24 \%$ obtiveram obstrução do fluxo aéreo. Dessa forma, baseados nesses resultados, sugerimos que as doenças parasitárias podem desenvolver, além das adaptações agudas, adaptações respiratórias crônicas.

Umas das limitações encontradas no presente estudo referem-se às características da amostra - composta por um indivíduo fumante, três ex-fumantes, dois com histórico de infecções respiratórias como 
tuberculose e asma brônquica e a existência de um indivíduo soropositivo. Inúmeros estudos apontam, no entanto, uma íntima relação entre a LV com condições que podem trazer prejuízo para a saúde pulmonar como condições sociais e econômicas desfavorecidas e a adoção de hábitos de vida prejudiciais, tais como o tabagismo e o etilismo (35), bem como a associação com outras doenças ditas oportunistas, as quais aproveitam a condição imunológica desfavorecida do indivíduo $(8,48)$. Dessa forma, a infecção por LV pode estar associada, em muitos casos, a condições desfavoráveis que podem, também, gerar adaptações na função pulmonar. Em pacientes com histórico de tabagismo, por exemplo, essas adaptações tendem a mostrar um padrão ventilatório obstrutivo (49); entretanto, a média dos resultados demonstrou tendência à restrição, sugerindo que tal padrão ventilatório poderia estar relacionado às adaptações crônicas pela LV.

\section{Conclusão}

Os dados obtidos por essa pesquisa evidenciaram uma relativa aproximação aos valores espirométricos que caracterizam um padrão ventilatório restritivo em indivíduos que sofreram previamente infecção por LV. Esses valores reforçam a possibilidade de um baixo desempenho na realização das manobras no teste de espirometria influenciando a saúde pulmonar dos indivíduos avaliados, visto que parece existir uma relação entre infestações parasitárias com alterações respiratórias estruturais. Apesar de o número de voluntários desta pesquisa ter sido pequeno, entre a maior parte dos indivíduos que se enquadravam nos critérios de seleção desta pesquisa foram encontradas diferenças altamente significativas.

A LV atualmente tornou-se uma preocupação da comunidade cientifica por incidir em mais de 60 países, colocando, portanto, o equivalente a 200 milhões de pessoas em risco de infecção, independentemente de cor, sexo ou condição socioeconômica (50). Nesse sentido, as comprovações dos nossos resultados reforçam a justificativa da necessidade de avaliações funcionais pulmonares após o período de infecção em indivíduos infectados por LV, com o objetivo de prevenir complicações respiratórias posteriores e encaminhar, conforme sua necessidade, a reabilitação cardiopulmonar, minimizando possíveis efeitos crônicos da LV e melhorando a qualidade de vida desses pacientes.

\section{Referências}

1. Desjeux P. Leishmaniasis: current situation and new perspectives. Comp Immunol Microbiol Infect Dis. 2004;27:305-18. doi:10.1016/j.cimid.2004.03.004.

2. Silva FS. Patologia e patogênese da leishmaniose visceral canina. Rev Trop Ciênc Agrár e Biol. 2007; 1(1):20-31.

3. Drumond KO, Costa FAL. Forty years of visceral leishmaniasis in the state of Piauí: a review. Rev Inst Med Trop. 2011;53(1):3-11. doi:10.1590/ S0036-46652011000100002.

4. Miranda GMD. Leishmaniose visceral em Pernambuco: a influência da urbanização e da desigualdade social [tese] Recife: Centro de Pesquisas Aggeu Magalhães; 2008.

5. Brasil. Ministério da Saúde. Manual de Vigilância e Controle da Leishmaniose Visceral. Brasília: Ministério da Saúde; 2006.

6. Braga ASC. Fatores associados à evolução clínica da leishmaniose visceral em crianças hospitalizadas em centro de referência de Belo Horizonte, 2001 a 2005 [dissertação]. Belo Horizonte: Faculdade de Medicina da Universidade Federal de Minas Gerais; 2007.

7. Neto JC, Werneck GL, Costa CHN. Factors associated with the incidence of urban visceral leishmaniasis: an ecological study in Teresina, Piauí State, Brazil. Cad Saúde Pública. 2009;25(7):1543-155. doi:10.1590/ S0102-311X2009000700012.

8. Batista IL, Sobrinho JA, Barros AAP, Nunes JC, Walber LA. A leishmaniose visceral como doença oportunista em um geronte portador da Síndrome da Imunodeficiência Adquirida (SIDA). Rev APS. 2004;7(2):119-24.

9. Lima BM, Batista RA. Epidemiologia da Leishmaniose Visceral Humana em Fortaleza-Ce. Rev Bras Promoção Saúde. 2009;22(1):16-23. doi:10.5020/ 18061230.2009.p16.

10. Almeida GF. Leishmanioses Visceral e Tegumentar Canina: Revisão da Literatura. [tese]. Campo Grande: Universidade Castelo Branco; 2006.

11. Darwazah AK, Hawari HM, Qaqa Z, Raed AH, Sham'a A, Sharabati B. Visceral leishmaniasis complicated by fungal pulmonary valve endocarditis. J Infect. 2006;53:185-89. doi:10.1016/j.jinf.2005.12.021. 
12. Silva ARS. Avaliação radiolográfica das articulações dos membros locomotores de cães naturalmente acometidos por Leishmaniose Visceral no município de Araçatuba-SP [dissertação]. São Paulo: Faculdade de Odontologia da Universidade Estadual Paulista; 2009.

13. Brasil. Ministério da Saúde. Manual de Vigilância e Controle da Leishmaniose Visceral. Brasília: Ministério da saúde; 2003. (Série A).

14. Bacellar O, Carvalho EM. Imunopatogênese da Leishmaniose Visceral. Gaz Méd Bahia. 2005; 75(1):24-34.

15. López-Peña M, Alemañ N, Muñoz F, Fondevila D, Suárez ML, Goicoa A, et al. Visceral leishmaniasis with cardiac involvement in a dog: a case report. Acta Vet Scand. 2009;51(20).

16. Alvarenga DG, Escalda PMF, Costa ASV, Monreal MTFD. Leishmaniose visceral: estudo retrospectivo de fatores associados à letalidade. Rev Soc Bras Med Trop. 2010;43(2):194-97. doi:10.1590/ S0037-86822010000200017.

17. Gonçalves R, Tafuri WL, Melo MN, Raso P, Tafuri WL. Chronic interstitial pneumonitis in dogs naturally infected with leishmania (leishmania) chagasi: a histopathological and morphometric study. Rev Inst Med Trop. 2003;45(3):153-58. doi:10.1590/ S0036-46652003000300007.

18. Alves GBB, Pinho FA, Silva SMMS, Cruz MSP, Costa FAL. Cardiac and pulmonary alterations in symptomatic and asymptomatic dogs infected naturally with Leishmania (Leishmania) chagasi. Braz J Med Biol Res. 2010;43(3):310-15. doi:10.1590/ S0100-879X2009007500037.

19. Casado JL, Cuesta C, Sanchez JA, Guerrero A. Solitary pulmonary nodule due to Leishmania in a patient with AIDS. Clin InfectDis. 1998;26(2):532-34. doi:10.1086/517084.

20. Tuon FF, Guedes F, Fernandes ER, Pagliari C, Amato VS, Seixas DMI. In situ immune responses to interstitial pneumonitis in human visceral leishmaniasis. Parasite Immunol. 2009;31:98-103. doi:10.1111/j.1365-3024.2008.01080.x.

21. Matheron S, Cabie A, Parquin F, Mayaud C, Roux $\mathrm{P}$, Antoine $\mathrm{M}$, et al. Visceral leishmaniasis and HIV infection: unusual presentation with pleuropulmonary involvement, and effect of secondary prophylaxis. AIDS. 1992;6:238-40. doi:10.1097/ 00002030-199202000-00020.
22. Chenoweth CE, Singal S, Pearson RD, Betts RF, Markovitz DM. Acquired immunodeficiency syndrome-related visceral leishmaniasis presenting in a pleural effusion [letter]. Chest. 1993;103:648-9. doi:10.1378/chest.103.2.648.

23. Herrejón A, Cervera A, Maciá M, Ferrer R, Blanquera R. Adenoma bronchioloalveolar associado à bronquiolite obliterante e leishmaniose com acometimento pulmonar na síndrome da imunodeficiência adquirida. Arch Bronconeumol. 2005;41(4):233-5. doi:10.1157/13073174.

24. Marshall BG, Kropf P, Murray K, Clark C, Flanagan AM, Davidson R, et al. Bronchopulmonary and Mediastinal Leishmaniasis: an unusual clinical presentation of leishmania donovani infection. Clin Infect Dis. 2000;30:764-9. doi:10.1086/313763.

25. Toledo AC Jr, Castro MR. Pneumocystis carinii Pneumonia, Pulmonary Tuberculosis and Visceral Leishmaniasis in an Adult HIV Negative Patient. Braz J Infect Dis. 2001;5(3):154-57. doi:10.1590/ S1413-86702001000300008.

26. Kotsifas K, Metaxas E, Koutsouvelis E, Skoutelis A, Kara $\mathrm{P}$, Tatsis G. Visceral leishmaniasis with endobronchial involvement in an immunocompetent adult. Case Reports in Medicine. 2011;1-5. doi:10.1155/ 2011/561985.

27. Romeu J, Sirera G, Ferrandiz C, Carreres A, Condom MJ, ClotetB. Visceral leishmaniasis involving lung and a cutaneous Kaposi's sarcoma lesion. AIDS. 1991;5(10):1272. doi:10.1097/ 00002030- 199110000-00025.

28. Nigro L, Montineri A, Rosa RL, Zuccarello M, Iacobello C, Iacobello C, et al. Visceral leishmaniasis and HIV co-infection: a rare case of pulmonary and oral localization. Infez Med. 2003;2:91-94.

29. Sundar S, Sinha P, Agrawal NK, Srivastava R, Rainey P, Berman JD, et al. A cluster of cases of severe cardiotoxicity among kala-azar patients treated with a high-osmolarity lot of sodium antimony gluconate. Am J Trop Med. 1998;59(1):139-43.

30. Paula CDR, Sampaio JHD, Cardoso DR, Sampaio RNR. Estudo comparativo da eficácia de isotionato de pentamidina administrada em três doses durante uma semana e de N-metil-glucamina $20 \mathrm{mgSbV} / \mathrm{kg} / \mathrm{dia}$ durante 20 dias para o tratamento da forma cutânea da leishmaniose tegumentar Americana. Rev Soc Bras Med Trop. 2003;36(3):65-371. doi:10.1590/ S0037-86822003000300009. 
31. Pereira CAC. Espirometria. J Pneumol. 2002;28(3 Suppl.):01-82.

32. ATS/ERS. Statement on respiratory muscle testing. Am J Respir Crit Care Med. 2002;(166):518-624.

33. Crapo RO, Morris AH, Gardner RM. Reference spirometric values using techniques and equipment that meet ATS recommendations. Am Rev Respir Dis. 1981; 23(6):659-64.

34. Neder JA, Andreoni S, Lerario MC, Nery LE. Reference values for lung function tests. II. Maximal respiratory pressures and voluntary ventilation. Braz J Med Biol Res. 1999;32:719-27. doi:10.1590/ S0100- 879X1999000600007.

35. Gontijo CMF, Melo MN. Leishmaniose visceral no Brasil: quadro atual, desafios e perspectivas. Rev Bras Epidemiol. 2004;7:338-49. doi:10.1590/ S1415-790X2004000300011.

36. Pedrosa CMS, Rocha EMM. Aspectos clínicos e epidemiológicos da leishmaniose visceral em menores de 15 anos procedentes de Alagoas, Brasil. Rev Soc Bras Med Trop. 2004;37(4):300-04. doi:10.1590/ S0037-86822004000400003.

37. Rey LC, Martins CV, Ribeiro HB, Lima AAM. Leishmaniose visceral americana (calazar) em crianças hospitalizadas de área endêmica. J Pediatr. 2005;81(1):73-8. doi:10.2223/JPED.1286.

38. Vargas FS, Cukier A, Neto MMR. Função pulmonar na esquistossomose mansoni. Arq Bras Cardiol. 1980; 3(2):60-62.

39. Manço JC, Terra J Filho. Comprometimento das vias aéreas na forma crônica da doença de chagas. J Pneumol. 1984;10(24):243-47.

40. Terra J Filho, Gallo L Jr, Maciel BC, Marin JÁ Neto, Manço JC. Estudo funcional das vias aéreas na forma crônica da doença de Chagas. J Bras Pneumol. 1986;12(2):91-100.

41. Botelho C, Guedes SB, Aquino JL, Silva MD, Meireles SMP, Jardim EJRB. Manifestações respiratórias na malaria por plasmodium falciparum e vivax. Rev Inst Med Trop. 1987;29:337-45. doi:10.1590/ S0036-46651987000600002.

42. Campos EP, Caetaneo AJM. Função pulmonar na evolução de 35 pacientes com Paracoccidioidomicose. Rev Inst Med Trop. 1986;28(5):330-36. doi:10.1590/ S0036-46651986000500008.
43. Martinez JAB. Doenças intersticiais pulmonares. Medicina. 1998;31:247-256.

44. Rodrigues SCS, Rodrigues MM, Colleta EMC, Rocha NS, Pereira CAC. Doença pulmonar intersticial associada à bronquiolite respiratória. J Bras Pneumol. 2004;30(6):574-80. doi:10.1590/ S1806-37132004000600013.

45. Duarte MIS, Laurenti MD, Nunes VLB, Rego AF Jr, Oshiro ET. Interstitial pneumonitis in canine visceral leishmaniasis. Rev Inst Med Trop. 1986; 28:431-36. doi:10.1590/S0036-46651986000600009.

46. Caneca OAF, Teixeira CB, Ladosky V, Almeida R. Alterações pulmonares cintilográficas e espirométricas em pacientes jovens portadores de Esquistossomose Mansônica tratados clínica e cirurgicamente na infância. Rev Col Bras Cir. 2002;29(5):26770. doi:10.1590/S0100-69912002000500004.

47. Park JS, Brown KK, Tuder RM, Hale VAE, King TE Jr, Lynch DA. Respiratory bronchiolitis-associated interstitial lung disease: radiologic features with clinical and pathologic correlation. J Comput Assist Tomogr. 2002;26:13-20. doi:10.1097/ 00004728-200201000-00003.

48. Furlan MBG. Epidemia de leishmaniose visceral no Município de Campo Grande-MS, 2002 a 2006. Epidemiol Serv Saúde. 2010;19(1):15-24.

49. Yaksic MS, Tojo M, Cukier A, Stelmach R. Perfil de uma população brasileira com doença pulmonar obstrutiva crônica grave. J Pneumol. 2003;29(2):64-8. doi:10.1590/S0102-35862003000200004.

50. Camizotti LA. Identificação e caracterização cromossomal de 9 loci de leishmania major relacionados com a resistência a inibidores da via biossintese do ergosterol [dissertação]. São Paulo: Faculdade de Medicina da Universidade de São Paulo; 2008.

Recebido: 29/07/2012

Received: 07/29/2012

Aprovado: 21/03/2013

Approved: 03/21/2013 